\title{
APPLICATION OF EXTENDED KALMAN FILTERING FOR ESTIMATING IMMEASURABLE VEHICLE STATE VARIABLES
}

\author{
Anh Tuan LE* \\ Faculty of Electrical and Electronics Engineering, Ton Duc Thang University, Ho Chi Minh City, \\ Vietnam \\ *leanhtuan1@tdt.edu.vn \\ (Received: 10-February-2017; accepted: 30-April-2017; published: 8-June-2017) \\ DOI: http://dx.doi.org/10.25073/jaec.201711.45
}

\begin{abstract}
This paper presents an extended Kalman filtering (EKF) algorithm for estimating immeasurable state variables of a vehicle stability control system. Initially, the steering angle and vertical forces on the tires were considered inputs of the estimator. The measured process outputs were the sensor signals regarding longitudinal and lateral accelerations, steering angle, yaw rate, and wheel speed. Subsequently, by using Euler discretization for a seven-degreeof-freedom nonlinear vehicle model, difficult-tomeasure state variables such as lateral velocity, vehicle side-slip angle, and lateral tire forces were identified separately by using the EKF algorithm. The estimation results of the proposed control system evidenced high performance.
\end{abstract}

\section{Keywords}

Extended Kalman filtering, Lateral force, Vehicle model, Side-slip angle, State variables.

\section{Introduction}

Modern intelligent automotive systems are equipped with a vehicle stability control (VSC), which is intended to enhance the active safety and handling predictability for drivers [1]. VSCs increase driver control on curves and slippery roads, substantially reducing the risk of fatal crashes. The operating principle of a VSC system is the use of differential braking mainly according to the exact steering angle, longitudinal velocity, yaw rate, vehicle side-slip angle, and tire slip angle to generate stabilizing yaw moment. In practice, longitudinal velocity can be measured according to wheel speed. The steering angle, yaw rate, and wheel speed can be determined using widely available and inexpensive sensors. The vehicle side-slip angle and tire slip angle can be derived if lateral tire forces and lateral velocity, respectively, are known. The sensors typically used to measure these variables are expensive. Therefore, different estimation methods for identifying these variables by using available low-cost sensors have been proposed. Mingli et al. (2010) presented kinematic integration to directly estimate the side-slip angle [2. However, this method cannot provide reliable estimation because of integration errors. Limroth (2009), Ungoren and Peng (2004), and Deng and Zhang (2006) used two states of the bicycle model, lateral velocity and the yaw rate, to design the observer identifying the lateral velocity [3]-5]. The bicycle model is based on assumptions such as a constant vehicle longitudinal velocity and small tire slip angle. These methods do not ensure exact estimation over all driving conditions because of model uncertainties. Zarchan and Musoff (2009) proposed a state and parameter estimator based on Kalman 
filtering (KF) theory, which has applications in many areas [6. Kalman (1960) proposed KF to estimate the immeasurable states in linear systems [7]. The fundamental operation of KF is a successive process of prediction based on system input, followed by correction based on measurable System output. An extension of KF theory developed for state estimation in nonlinear systems is named the extended Kalman filtering (EKF) algorithm. The main concept is the same as that of standard KF, but the computational complexity is increased.

Two approaches are employed for EKF. The first approach is that of an optimization algorithm that recursively determines states to minimize a cost function. The second approach is that of EKF providing approximate maximumlikelihood estimates. Similar to the first interpretation, the mean and covariance of the states are recursively updated. However, a firstorder linearization of the dynamics is necessary to analytically propagate the Gaussian randomvariable representation. Effectively, the nonlinear dynamics are approximated by a timevarying linear system, and linear KF equations are applied.

Chen and Hsieh (2008) proposed vehicle sideslip angle estimator based on the kinematics of the simpliest modified bicycle model. The EKF was used to design the estimation gain. For this approach, the lateral tire forces were not estimated. Moreover, the kinematic model was derived from lateral and longitudinal kinematic equations with the pole placement approach [8]. These equations are sometimes not sufficient for describing whole vehicle motion because of the motion's high nonlinearity. Parameter uncertainties might result in estimation errors. In this study, an EKF algorithm was applied to identify difficult-to-measure vehicle variables. It was derived basing on a seven-degree-of-freedom (7-DOF) four-wheel nonlinear vehicle model, instead of bicycle model [8]. By using the proposed method, the lateral velocity, vehicle sideslip angle, and lateral forces of each tire were estimated reliably. Consequently, the results of this study can be used to obtain side-slip angle and lateral tire forces by using low-cost sensors of steering angle, yaw rate, wheel speed, and lat- eral and longitudinal accelerations. In practice, these sensors are readily available. Compared with my previous study [9], firstly, the verification of vehicle model was added in proposed study for its reliability in estimation algorithm. Secondly, from the information of wheel speed sensors, the forward speed can be identified. The EKF estimation algorithm exists in the conditions that vehicle is not at rest and the steering angle is not equal to zero. The EKF algorithm in the previous paper disregarded a case that the steering angle of zero means the tire sideslip angles of zero. Simultaneously, the lateral forces of the four wheels are also equal to zero. Therefore, the estimation error of side-slip angle became high for the steering angle of zero in previous paper. In this study, the vehicle side-slip angle can be estimated favorably with all conditions of the steering angle. Thirdly, the parameters of the proposed EKF algorithm were also set and analyzed. Fourthly, the simulation time for steering angle was longer than that of previous study. The goal was to test whether the estimation of vehicle side-slip angle is always kept favorably. Moreover, the vertical force input of the EKF was also computed and compared with that from CarSim. And finally, the esimation results were discussed in detail by calculating the root mean square error (RMSE) values for lateral tire forces and the vehicle side-slip angle. They were analyzed according to different cases of steering angle.

This paper comprises five parts. The motivation of the study is presented in Section 1. In Section 2, the 7-DOF vehicle and tire models for the estimator design are described. The method for estimating immeasurable vehicle state variables is proposed in Section 3. Section 4 presents the simulation results. Finally, conclusions are presented in Section 5, summarizing the main points of the paper and recommending future research directions.

\section{Vehicle System}

\subsection{Vehicle Model}

When applying the EKF algorithm for estimating immeasurable variables in a VSC system, 
the dynamics of the vehicle must be precisely defined. As a result, the derived vehicle model must be compared with a standard model to ensure its reliability.

The CarSim vehicle model, a high-order model covering all degrees of freedom (18 DOF) of a vehicle, is a commercially available product with a well-defined user interface that can verify the accuracy of a built model.

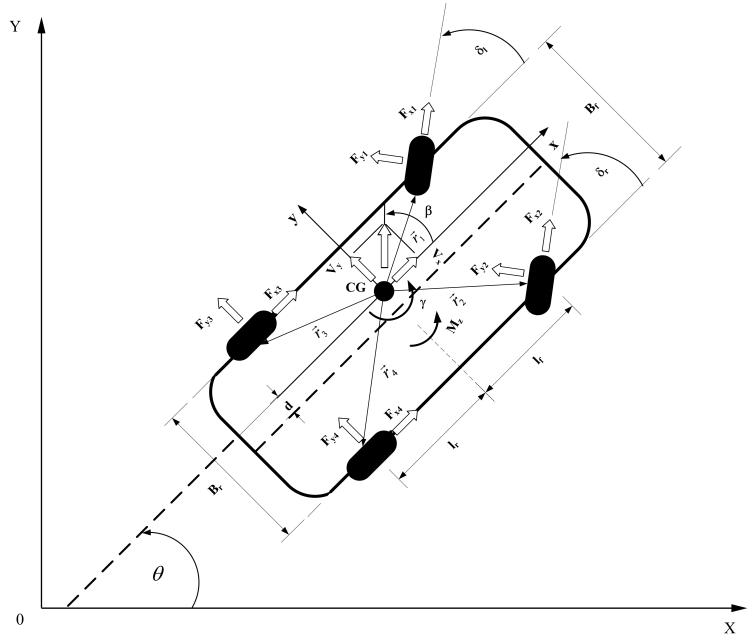

Fig. 1: 7-DOF vehicle model.

Demerly and Toumi (2000) reduced the order of a CarSim model to a vehicle model with eight degrees of freedom [10]. This model consisted of longitudinal and lateral velocities originating from the chassis, the vertical body roll angle, the vehicle yaw rate, and the angular velocities of the four wheels. For simplicity in the estimator design, the current study constructed a 7-DOF vehicle model that disregarded the roll dynamics (Fig. 1). The governing equations of motion (EOMs) are derived by applying the NewtonEuler approach to the wheels and vehicle as follows:

Longitudinal dynamics

$$
\begin{gathered}
\dot{V}_{x}=\frac{1}{m}\left(m \gamma V_{y}+F_{x 1} \cos \delta_{l}+F_{x 2} \cos \delta_{r}+\right. \\
\left.F_{x 3}+F_{x 4}-F_{y 1} \sin \delta_{l}-F_{y 2} \sin \delta_{r}\right)
\end{gathered}
$$

Lateral dynamics

$$
\begin{gathered}
\dot{V}_{y}=\frac{1}{m}\left(-m \gamma V_{x}+F_{x 1} \sin \delta_{l}+F_{x 2} \sin \delta_{r}+\right. \\
\left.F_{y 3}+F_{y 4}+F_{y 1} \cos \delta_{l}+F_{y 2} \cos \delta_{r}\right)
\end{gathered}
$$

Yaw dynamics

$$
\begin{aligned}
& \dot{\gamma}=\frac{1}{I_{z}}\left(F_{y 1}\left(l_{f} \cos \delta_{l}+A_{a f} \sin \delta_{l}\right)-\left(F_{y 3}\right.\right. \\
& \left.+F_{y 4}\right) l_{r}+F_{y 2}\left(l_{f} \cos \delta_{r}-B_{b f} \sin \delta_{r}\right) \\
& +F_{x 1}\left(-A_{a f} \cos \delta_{l}+l_{f} \sin \delta_{l}\right)+F_{x 2}\left(l_{f} \sin \delta_{r}+\right. \\
& \left.\left.B_{b f} \cos \delta_{r}\right)-F_{x 3} A_{a r}+F_{x 4} B_{b r}\right)
\end{aligned}
$$

Rotational dynamics of the four wheels

$$
\dot{\omega}_{n}=\frac{1}{I_{w}}\left(r F_{x n}-T_{b n}\right), \quad(n=1,4)
$$

where

$$
\begin{array}{ll}
A_{a f}=0.5 B_{f}-d ; & A_{a r}=0.5 B_{r}-d \\
B_{b f}=0.5 B_{f}+d ; & B_{b r}=0.5 B_{r}+d
\end{array}
$$

\subsection{Tire Model}

The Dugoff tire model which was adopted from [11] is used to represent nonlinear tire behavior because of its small number of parameters, which are sufficient for evaluating tire-road forces. Both the longitudinal and lateral forces of each tire can be expressed as

$$
\begin{aligned}
& F_{x n}=C_{d n} \frac{i_{d n}}{1+i_{d n}} f\left(\lambda_{n}\right) \\
& \bar{F}_{y n}=-C_{n} \frac{\tan \alpha_{n}}{1+i_{d n}} f\left(\lambda_{n}\right)
\end{aligned}
$$

where

$$
\bar{F}_{y n}=-C_{n} \frac{\tan \alpha_{n}}{1+i_{d n}} f\left(\lambda_{n}\right)
$$

The slip angles of the front and rear tires can be obtained using

$$
\begin{gathered}
\alpha_{1}=\delta_{l}-\arctan \left(\frac{V_{y}+l_{f} \gamma}{V_{x}-\left(0.5 B_{f}-d\right) \gamma}\right) \\
\alpha_{2}=\delta_{r}-\arctan \left(\frac{V_{y}+l_{f} \gamma}{V_{x}+\left(0.5 B_{f}+d\right) \gamma}\right)
\end{gathered}
$$



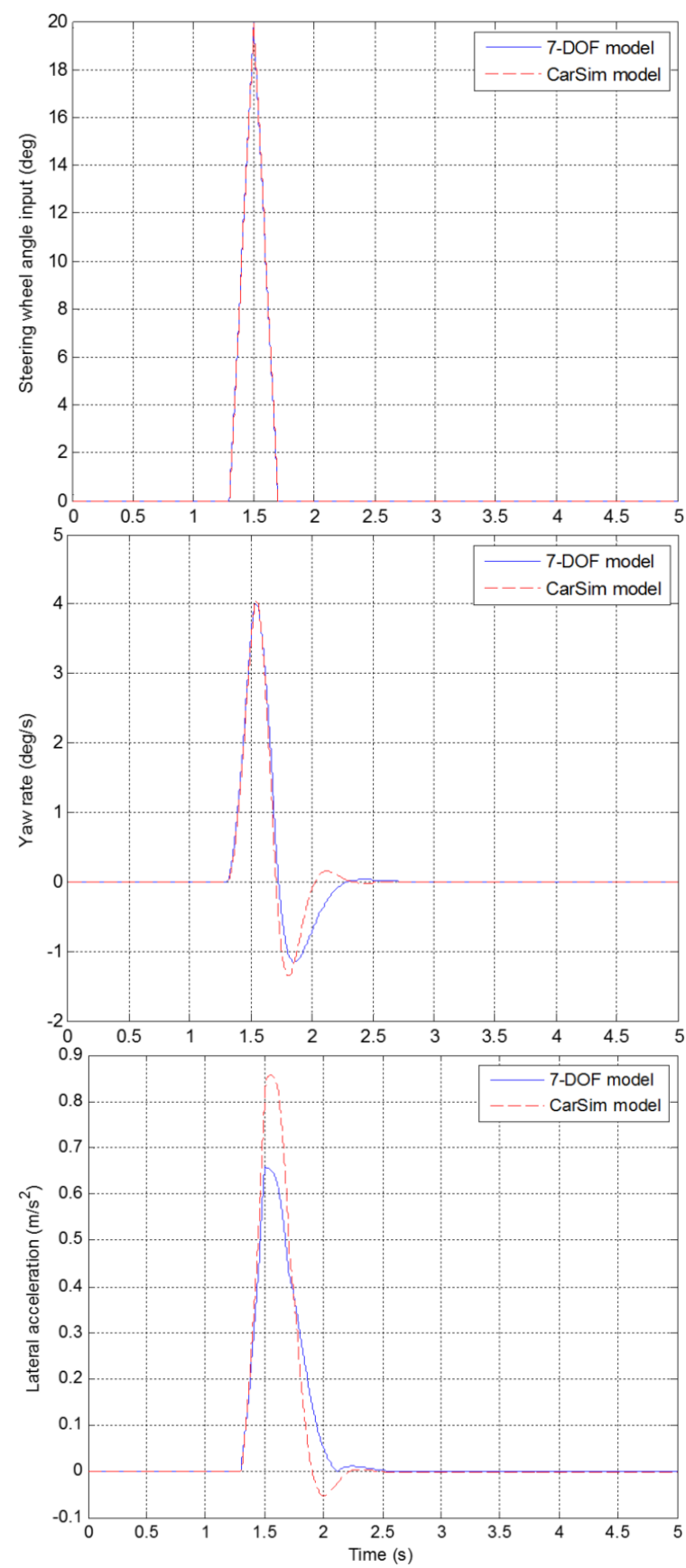

Fig. 2: Yaw rate and lateral acceleration responses.

$$
\begin{aligned}
& \alpha_{3}=-\arctan \left(\frac{V_{y}-l_{r} \gamma}{V_{x}-\left(0.5 B_{r}-d\right) \gamma}\right) \\
& \alpha_{4}=-\arctan \left(\frac{V_{y}-l_{r} \gamma}{V_{x}+\left(0.5 B_{r}+d\right) \gamma}\right)
\end{aligned}
$$

According to [11, lag exists between a sudden change in the slip angle of each tire and the buildup of a corresponding lateral force. This lag can be modeled as

$$
\dot{F}_{y n}=\frac{V_{x}}{\varepsilon}\left(-F_{y n}+\bar{F}_{y n}\right)
$$

where $F_{y n}$ is the lagged lateral force of each tire and $\varepsilon$ is the relaxation length.

\subsection{Vehicle-model Verification}

As discussed previously, although the vehicle model has reduced DOF, it can approximate the results of the high-order model in some situations. The yaw rate and lateral acceleration responses of two compared models are illustrated in Fig. 2,

According to [12], the steering wheel angle input is a triangular pulse with a pulse width of 0.4 $\mathrm{s}$ in the two models. Dugoff's tire model enables calculating longitudinal and lateral forces in a closed form, which is required for implementing the simulation. The vehicle simulation parameters were described in the previous studies [1] and $[13$.

\section{Extended Kalman Filtering}

\subsection{Nonlinear state-space model}

To avoid the computational burden of deriving the EKF algorithm applied to the 7-DOF vehicle model in this study, the vehicle model was simplified using the following assumptions:

Under the supposition that $\mathrm{d}=0$, Eqs. (4) and (5) become

$$
A_{a f}=B_{b f}=0.5 B_{f}, \quad A_{a r}=B_{b r}=0.5 B_{r}
$$

Subsequently, it is supposed that

$$
\delta_{l} \approx \delta_{r} \approx \delta, \quad F_{x 3} \approx F_{x 4} \approx 0
$$

Setting

$$
F_{x 1}+F_{x 2}=F_{x 11}, \quad F_{x 2}-F_{x 1}=F_{x 12}
$$


The EOMs Eqs. (1)-(3) become

$$
\begin{gathered}
\dot{V}_{x}=\frac{1}{m}\left(m \gamma V_{y}+F_{x 11} \cos \delta\right. \\
\left.-\left(F_{y 1}+F_{y 2}\right) \sin \delta\right) \\
\dot{V}_{y}=\frac{1}{m}\left(-m \gamma V_{x}+F_{x 11} \sin \delta\right. \\
\left.+\left(F_{y 1}+F_{y 2}\right) \cos \delta+F_{y 3}+F_{y 4}\right) \\
\dot{\gamma}=\frac{1}{I_{z}}\left(l_{f} F_{x 11} \sin \delta+l_{f}\left(F_{y 1}+F_{y 2}\right) \cos \delta\right. \\
-l_{r}\left(F_{y 3}+F_{y 4}\right)+0.5 B_{f} F_{x 12} \cos \delta \\
\left.+0.5 B_{f}\left(F_{y 1}-F_{y 2}\right) \sin \delta\right)
\end{gathered}
$$

and the slip angles Eqs. (9)- 12 are similar to

$$
\begin{gathered}
\alpha_{1}=\delta-\arctan \left(\frac{V_{y}+l_{f} \gamma}{V_{x}-0.5 B_{f} \gamma}\right) \\
\alpha_{2}=\delta-\arctan \left(\frac{V_{y}+l_{f} \gamma}{V_{x}+0.5 B_{f} \gamma}\right) \\
\alpha_{3}=-\arctan \left(\frac{V_{y}-l_{r} \gamma}{V_{x}-0.5 B_{r} \gamma}\right) \\
\alpha_{4}=-\arctan \left(\frac{V_{y}-l_{r} \gamma}{V_{x}+0.5 B_{r} \gamma}\right)
\end{gathered}
$$

From Eqs. (14) and (15), the longitudinal and lateral accelerations are derived as follows:

$$
\begin{aligned}
a_{x} & =\dot{V}_{x}-\gamma V_{y} \\
& =\frac{1}{m}\left(F_{x 11} \cos \delta-\left(F_{y 1}+F_{y 2}\right) \sin \delta\right) \\
a_{y} & =\dot{V}_{y}+\gamma V_{x}=\frac{1}{m}\left(F_{x 11} \sin \delta\right. \\
& \left.+\left(F_{y 1}+F_{y 2}\right) \cos \delta+F_{y 3}+F_{y 4}\right)
\end{aligned}
$$

According to Eq. (21), the lateral dynamics 15. can be obtained in an alternative manner, namely by using

$$
\dot{V}_{y}=-\gamma V_{x}+a_{y}
$$

During EKF calculation, Eq. 22 should be prioritized over (15) because the yaw rate $\gamma$, longitudinal speed $V_{x}$, and lateral acceleration $a_{y}$ can be measured using the available sensors, yielding an accurate lateral velocity estimate.

Taking the time derivative of 22 yields

$$
\ddot{V}_{y}=\dot{a}_{y}-\gamma \dot{V}_{x}-\dot{\gamma} V_{x}
$$

where

$$
\ddot{a}_{y}=0
$$

In addition, with the suppositions that

$$
\begin{aligned}
& \dot{F}_{x 11} \approx 0 \\
& \dot{F}_{x 12} \approx 0
\end{aligned}
$$

Subsequently, considering the longitudinal skid ratio of each tire as zero $\left(i_{d n}=0\right)$, Eqs. (6)- (8) are similar to

$$
\begin{gathered}
F_{x n}=0 \\
\bar{F}_{y n}=-\left(C_{n} \tan \alpha_{n}\right) f\left(\lambda_{n}\right)
\end{gathered}
$$

where

$$
f\left(\lambda_{n}\right)=\left\{\begin{array}{cc}
\left(2-\lambda_{n}\right) \lambda_{n} & \text { if } \lambda_{n}<1 \\
1 & \text { if } \lambda_{n} \geqslant 1
\end{array}\right.
$$

and

$$
\lambda_{n}=\frac{\mu F_{z n}}{2 C_{n} \tan \alpha_{n}}
$$

Therefore, the dynamic tire model 13 becomes

$$
\dot{F}_{y n}=\frac{V_{x}}{\varepsilon}\left(-F_{y n}-\left(C_{n} \tan \alpha_{n}\right) f\left(\lambda_{n}\right)\right)
$$

where $f\left(\lambda_{n}\right)$ is obtained from Eqs. (27) and (28), and $\tan \alpha_{n}(n=1,4)$ is calculated by changing Eqs. 17 -20 as follows:

$$
\begin{gathered}
\tan \alpha_{1}=\frac{\left(V_{x}-0.5 B_{f} \gamma\right) \tan \delta-V_{y}-l_{f} \gamma}{V_{x}-0.5 B_{f} \gamma+\left(V_{y}+l_{f} \gamma\right) \tan \delta} \\
\tan \alpha_{2}=\frac{\left(V_{x}+0.5 B_{f} \gamma\right) \tan \delta-V_{y}-l_{f} \gamma}{V_{x}+0.5 B_{f} \gamma+\left(V_{y}+l_{f} \gamma\right) \tan \delta} \\
\tan \alpha_{3}=\frac{l_{r} \gamma-V_{y}}{V_{x}-0.5 B_{r} \gamma} \\
\tan \alpha_{4}=\frac{l_{r} \gamma-V_{y}}{V_{x}+0.5 B_{r} \gamma}
\end{gathered}
$$

Eqs. (14)-(16), (23)-(26) and (29) can be considered the following general state differential equation:

$$
\dot{\boldsymbol{x}}(t)=f[\boldsymbol{x}(t), \boldsymbol{u}(t), t]
$$

According to [14, applying Euler discretization to 34 yields

$$
\hat{\boldsymbol{x}}_{k \mid k-1}=\hat{\boldsymbol{x}}_{k-1 \mid k-1}+\hat{\dot{\boldsymbol{x}}}_{k-1 \mid k-1} T_{s}
$$


where $T_{s}$ is the sampling time and the derivative is obtained from

$$
\hat{\dot{\boldsymbol{x}}}_{k-1 \mid k-1}=f\left(\hat{\boldsymbol{x}}_{k-1 \mid k-1}, \boldsymbol{u}_{k}, t_{k}, t_{k-1}\right)
$$

The nonlinear discrete-time process of the whole vehicle system can then be rewritten in the following standard state-space form:

$$
\left\{\begin{array}{l}
\hat{\boldsymbol{x}}_{k \mid k-1}=\boldsymbol{f}\left(\hat{\boldsymbol{x}}_{k-1 \mid k-1}, \boldsymbol{u}_{k}, t_{k}, t_{k-1}\right)+\boldsymbol{w}_{k-1} \\
\tilde{\boldsymbol{y}}_{k}=\hat{\boldsymbol{y}}_{k}+\boldsymbol{v}_{k}
\end{array}\right.
$$

where $\boldsymbol{w}_{k-1}$ and $\boldsymbol{v}_{k}$ are respectively the process and measurement noises, and

$$
\hat{\boldsymbol{y}}_{k}=h\left(\hat{\boldsymbol{x}}_{k \mid k-1}, \boldsymbol{u}_{k}, t_{k}\right)
$$

The states of the process are expressed as

$$
\begin{aligned}
& \hat{\boldsymbol{x}}_{k \mid k-1}=\left[\begin{array}{lll}
\hat{V}_{x, k \mid k-1} & \hat{V}_{y, k \mid k-1} & \hat{\gamma}_{k \mid k-1}
\end{array}\right. \\
& \hat{F}_{y 1, k \mid k-1} \quad \hat{F}_{y 2, k \mid k-1} \quad \hat{F}_{y 3, k \mid k-1} \\
& \hat{F}_{y 4, k \mid k-1} \quad \hat{F}_{x 11, k \mid k-1} \quad \hat{F}_{x 12, k \mid k-1} \\
& \left.\hat{\dot{V}}_{y, k \mid k-1} \quad \hat{\dot{a}}_{y, k \mid k-1}\right]
\end{aligned}
$$

The vector of inputs is

$$
\boldsymbol{u}_{k}=\left[\begin{array}{lllll}
\delta_{k} & F_{z 1, k} & F_{z 2, k} & F_{z 3, k} & F_{z 4, k}
\end{array}\right]
$$

and the vector of the measured process outputs is

$$
\tilde{\boldsymbol{y}}_{k}=\left[\begin{array}{cccc}
\tilde{V}_{x, k} & \tilde{\gamma}_{k} & \tilde{a}_{x, k} & \tilde{a}_{y, k}
\end{array}\right]
$$

\subsection{Application of Extended Kalman Filtering}

For applying the EKF algorithm, it is assumed that the process and measurement noises are zero-mean Gaussian processes.

The process and measurement noise autocovariance matrices of $\boldsymbol{w}_{k-1}$ and $\boldsymbol{v}_{k}$ must be specified as follows:

$$
\begin{aligned}
\boldsymbol{Q}_{k, k-1} & =\boldsymbol{E}\left\{\boldsymbol{w}_{k-1} \cdot \boldsymbol{w}_{k-1}^{T}\right\} \\
\mathbf{R}_{k} & =\boldsymbol{E}\left\{\boldsymbol{v}_{k} \cdot \boldsymbol{v}_{k}^{T}\right\}
\end{aligned}
$$

The extent of the EKF algorithm bandwidth and its susceptibility to sensor measurement noises completely depend on its autocovariance matrix of process noise $\boldsymbol{Q}_{k, k-1}$, which represents

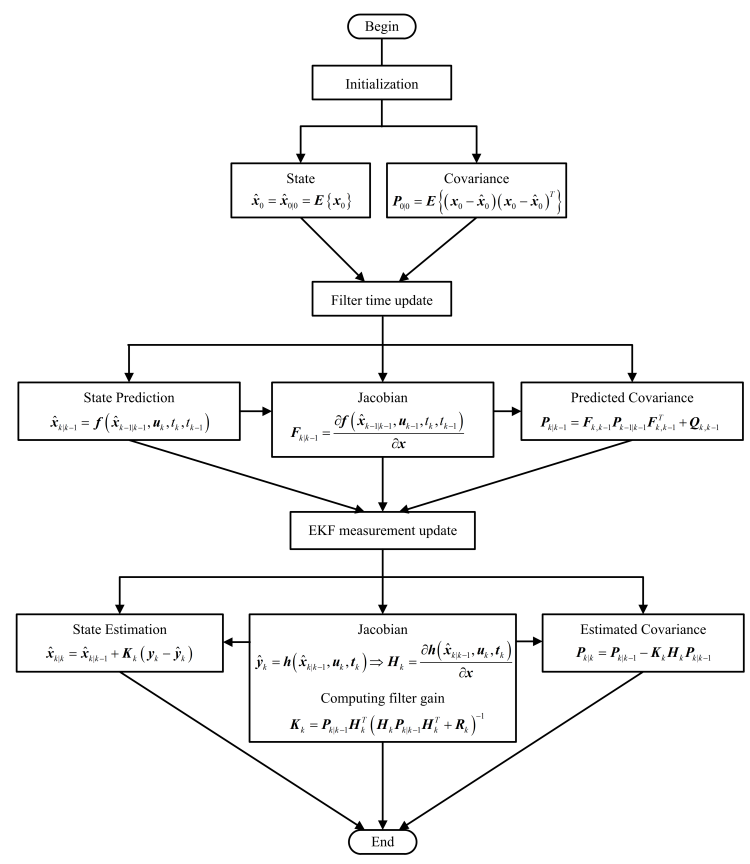

Fig. 3: Flow chart of EKF algorithm implementation.

the level of confidence placed in the accuracy of the observer model, and the autocovariance matrix of measurement noise $\boldsymbol{R}_{k}$, which represents the level of confidence placed in the accuracy of the sensor measurements. These values are used to tune the filter characteristics, including the accuracy and response, and were experimentally determined using sensor measurements. The EKF algorithm is described using a flow chart (Fig. 3). The input vector in the algorithm comprises the steering angle and vertical forces on the tires. According to [15], these forces can be approximately calculated by using

$$
\begin{aligned}
& F_{z 1}=\frac{m}{l}\left(\frac{g l_{r}}{2}-\frac{h_{c} a_{x}}{2}-\frac{h_{c} l_{r} a_{y}}{B_{f}}+\frac{h_{c}^{2} a_{x} a_{y}}{B_{f} g}\right) \\
& F_{z 2}=\frac{m}{l}\left(\frac{g l_{r}}{2}-\frac{h_{c} a_{x}}{2}+\frac{h_{c} l_{r} a_{y}}{B_{f}}-\frac{h_{c}^{2} a_{x} a_{y}}{B_{f} g}\right) \\
& F_{z 3}=\frac{m}{l}\left(\frac{g l_{f}}{2}+\frac{h_{c} a_{x}}{2}-\frac{h_{c} l_{f} a_{y}}{B_{r}}-\frac{h_{c}^{2} a_{x} a_{y}}{B_{r} g}\right) \\
& F_{z 4}=\frac{m}{l}\left(\frac{g l_{f}}{2}+\frac{h_{c} a_{x}}{2}+\frac{h_{c} l_{f} a_{y}}{B_{r}}+\frac{h_{c}^{2} a_{x} a_{y}}{B_{r} g}\right)
\end{aligned}
$$


where $h_{c}$ is the height of the center of gravity (CG). The vertical forces from Eqs. (35)-(38) can be measured using the information from the longitudinal and lateral acceleration sensors.

\section{Simulation Results}

Simulations were conducted in the MATLAB and Simulink environment and linked to the CarSim software. The CarSim vehicle model was a full sedan; the parameters of its tires and aerodynamics, such as sprung mass, powertrain, and suspension, were shown in my previous study [1].

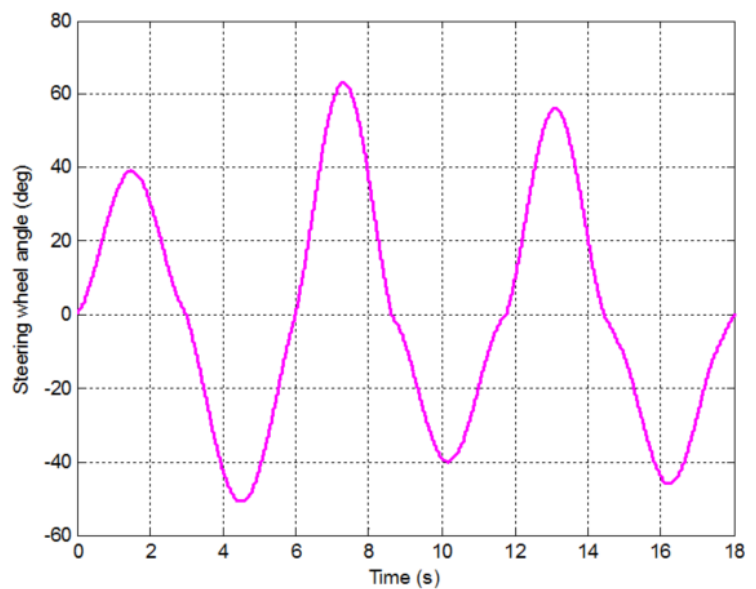

Fig. 4: Steering wheel angle input $(\delta \neq 0)$.

In this study, the process and measurement noise autocovariance matrices were selected as follows:

$$
\begin{gathered}
\boldsymbol{Q}_{k, k-1}=\operatorname{diag}\left[Q_{\hat{V}_{x}}, Q_{\hat{V}_{y}}, Q_{\hat{\gamma}}, Q_{\hat{F}_{y 1}}, Q_{\hat{F}_{y 2}},\right. \\
\left.Q_{\hat{F}_{y 3}}, Q_{\hat{F}_{y 4}}, Q_{\hat{F}_{x 11}}, Q_{\hat{F}_{x 12}}, Q_{\hat{V}_{y}}, Q_{\hat{\dot{a}}_{y}}\right] \\
\boldsymbol{R}_{k}=\kappa T_{s} \boldsymbol{I}
\end{gathered}
$$

where $\boldsymbol{I}$ is the unit matrix and $\kappa$ is a low positive constant selected to adjust the estimation results.

Treating the noise autocovariance matrices as a diagonal matrix was advantageous because it reduced computation time. For the selection of autocovariance matrices, it should be noted that the less the noise in sensor measurements compared with the uncertainty in the dynamics model, the more the states are suited to follow sensor measurements. The vehicle was assumed to be driven on a road with a steering input that changed according to the steering wheel angle, similar to that shown in Fig. 4 .
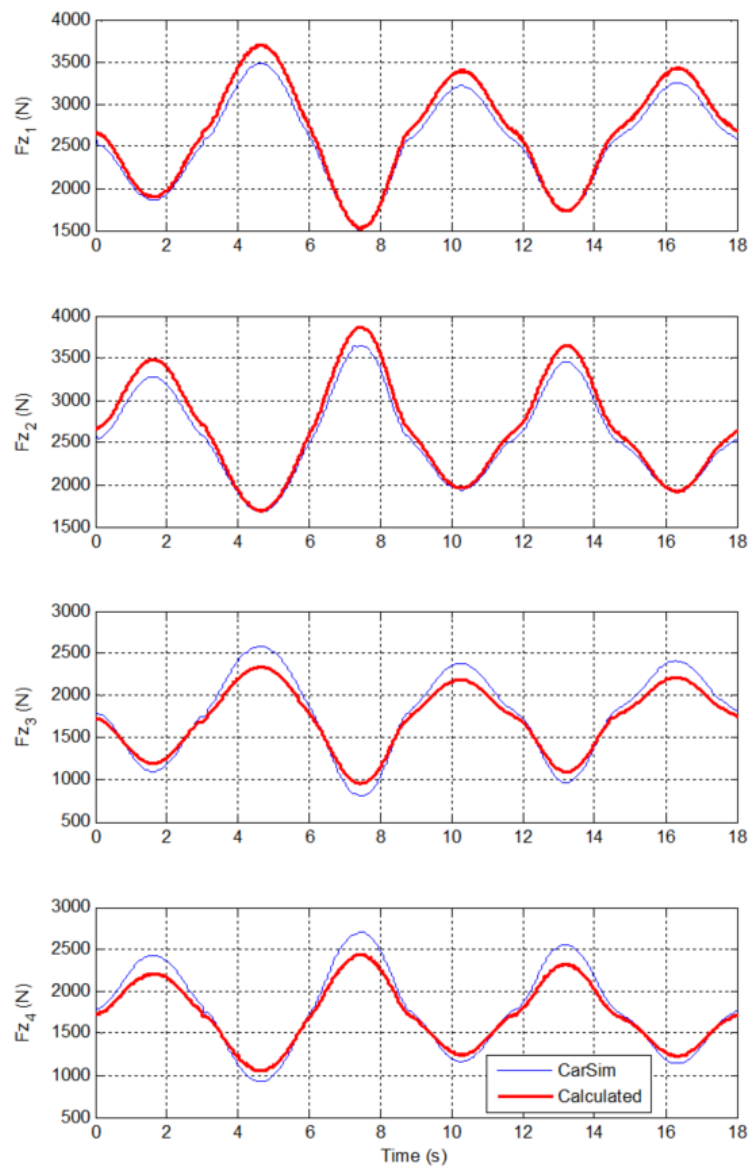

Fig. 5: Vertical forces from CarSim and calculation.

Initially, based on the measurable signals of longitudinal and lateral accelerations, the vertical forces were approximately calculated using Eqs. (35)-(38). Subsequently, these forces were compared with the standard vertical forces of the CarSim vehicle model as shown in Fig. 5 Eqs. (35)-(38) were derived without considering the camber angle and suspension dynamics. The roll and pitch accelerations were not considered. Furthermore, the roll and pitch axes were assumed to pass through the CG. Consequently, in Fig. 5 errors existed between the calculated vertical forces from Eqs. (35)- 38 ) and the vertical 
forces from the CarSim vehicle model. However, slip angle, using the following expression: these errors were acceptable.
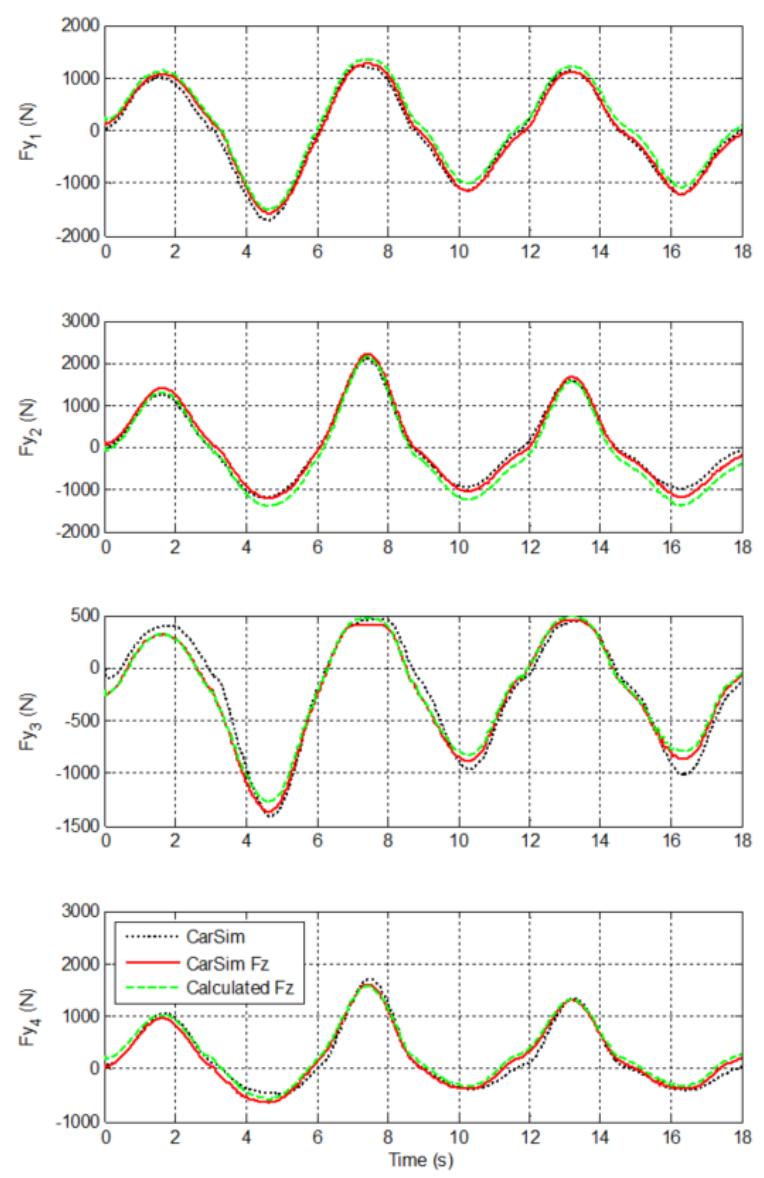

Fig. 6: Estimation of the four-wheel-lateral-tire forces.

Each estimate (Figs. 6 8) contained the output responses of lateral forces, lateral velocity, and the side-slip angle from the CarSim vehicle model as the standard signals for the estimator performance evaluation; lateral forces, lateral velocity, and the side-slip angle from the estimator when its vertical-force inputs were directly obtained from the CarSim vehicle model; and lateral forces, lateral velocity, and the side-slip angle from the estimator when its vertical-force inputs were calculated using Eqs. (35)-(38). Because differences in the estimator performance levels were difficult to observe in Figs. 6 and 8 they were compared by calculating the RMSE values for lateral tire forces and the vehicle side-

$$
R M S E=\left(\frac{1}{N} \sum_{k=1}^{N} e(k)^{2}\right)^{1 / 2}
$$

where $N$ is the number of samples. In addition, $e(k)$ can refer to either the estimation error of lateral tire forces $e_{F_{y n}}$ or the estimation error of the side-slip angle $e_{\beta}$. These errors were obtained as follows:

$$
\begin{gathered}
e_{F_{y n}}=F_{y n e s t}-F_{y n c a r}, \quad(n=1,4) \\
e_{\beta}=\beta_{\text {est }}-\beta_{c a r}
\end{gathered}
$$

where $F_{\text {ynest }}$ and $\beta_{\text {est }}$ are either the lateral forces and the side-slip angle, respectively, estimated when the EKF algorithm inputs were the vertical forces $F_{z n}$ as computed by using Eqs. (35)(38), or the lateral forces and side-slip angle, respectively, estimated when the EKF algorithm inputs were the vertical forces from the CarSim vehicle model. In addition, $F_{y n c a r}$ and $\beta_{c a r}$ are the standard signals of the lateral forces and side-slip angle from the CarSim vehicle model. The results are reported in Table 1 .

Tab. 1: Comparison among the estimation results obtained using different vertical force inputs.

\begin{tabular}{|l|l|l|}
\hline$R M S E s($ Unit $)$ & $F_{z i}$ from CarSim & Calculated $F_{z i}$ \\
\hline$R M S e_{F y 1}(N)$ & 90 & 152 \\
\hline$R M S e_{F y 2}(N)$ & 109 & 210 \\
\hline$R M S e_{F y 3}(N)$ & 97 & 110 \\
\hline$R M S e_{F y 4}(N)$ & 104 & 126 \\
\hline$R M S e_{b}($ rad $)$ & 0.0523 & 0.0939 \\
\hline
\end{tabular}

Figs. 648 and Fig. 10 present the results of the simulation with road and driving conditions for which the tire-road frictional coefficient was 0.85 and vehicle longitudinal velocity was 100 $\mathrm{km} / \mathrm{h}$. In all the simulations, using the estimator with vertical-force inputs directly obtained from the CarSim vehicle model yielded the optimal estimation results. The RMSE values for all estimations (Table 1) indicated that using the EKF algorithm inputs from the CarSim vehicle model as vertical forces always resulted in a more favorable performance than did using the EKF algorithm inputs calculated using Eqs. (35)-(38) as vertical forces. In particular, the RMSE values were low when using vertical forces calculated from Eqs. (35)-(38) and lower when using 
vertical forces from the CarSim vehicle model. Therefore, the applied EKF algorithm was extremely effective in all estimations when the vertical forces were modeled correctly. The first lateral force estimates (Fig. 6) were relatively favorable and exhibited acceptable errors. The EKF algorithm linearized the state and measurement equations neighboring the predicted state as an operating point. This may be one reason for the errors in the lateral force estimation. Other reasons may be that the rear longitudinal tire forces were assumed to be zero in estimator design but were not zero in practice. The lateral tire forces were affected by the camber angle, which was neglected in the vehicle model. In addition, errors existed in Eqs. (35)-(38), namely in the calculation of the vertical forces, which were part of the estimator input. Longitudinal velocity $V_{x}$ can be measured by the wheel speed sensors. In particular, the relationship of longitudinal velocity and wheel speed variables was approximately computed as follows:

$$
V_{x}=\frac{1}{4} \sum V_{n}, \quad(n=1,4)
$$

where ground contact point velocity of each wheel is

$$
V_{n}=r \omega_{n}, \quad(n=1,4)
$$

The lateral velocity can be obtained using the EKF estimation algorithm in the simulation (Fig. 7).

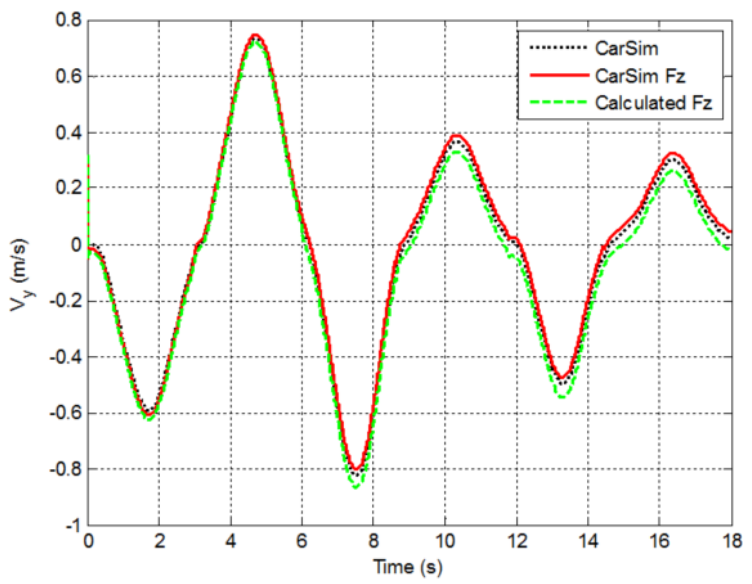

Fig. 7: Estimation of the lateral velocity.

In addition, the lateral velocity was also the basis for estimating the vehicle side-slip angle by

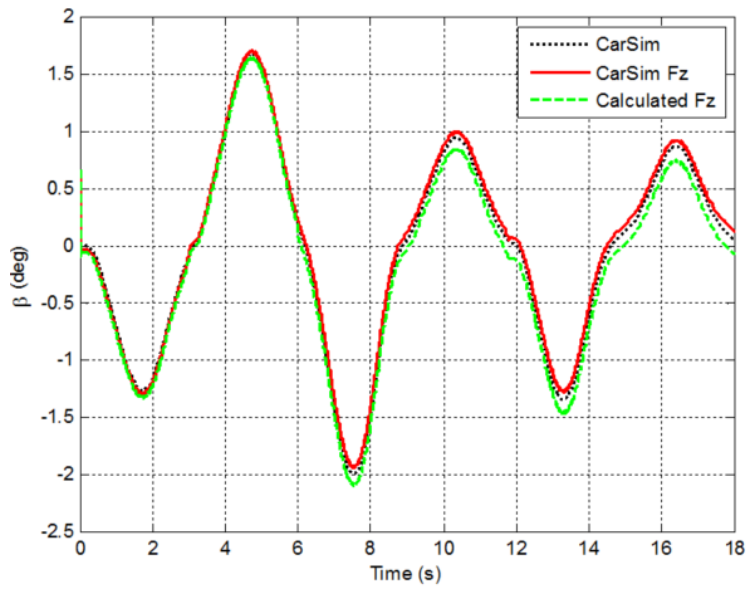

Fig. 8: Estimation of the vehicle side-slip angle for steering angle $\delta \neq 0$.

using

$$
\hat{\beta}=\arctan \left(\frac{\hat{V}_{y}}{V_{x}}\right)
$$

The second estimate of the lateral velocity (Fig. 7) matched the CarSim standard value almost exactly. This was because of the prior consideration of the usage between lateral dynamics from Eqs. (15) and (22) while implementing the EKF algorithm. The final side-slip angle estimate derived from equation (39) was favorable, being highly similar to its real value in the CarSim model (Fig. 8). To test whether the estimation result of vehicle side-slip angle remained favorable for any situation of steering angle $(\delta=0$ or $\delta \neq 0)$. The vehicle was assumed to be driven on a road with a steering input shown in Fig. 9. Fig. 10 showed that the estimation result was sustained satisfactorily when the steering angle began to change to zero and vice versa.

\section{Conclusions}

This study involved applying the EKF algorithm to estimation of immeasurable vehicle state variables, namely lateral velocity, vehicle side-slip angle, and lateral forces. The simulation results show if the vertical forces are calculated correctly and the EKF parameters are selected properly, the estimation performances are im- 


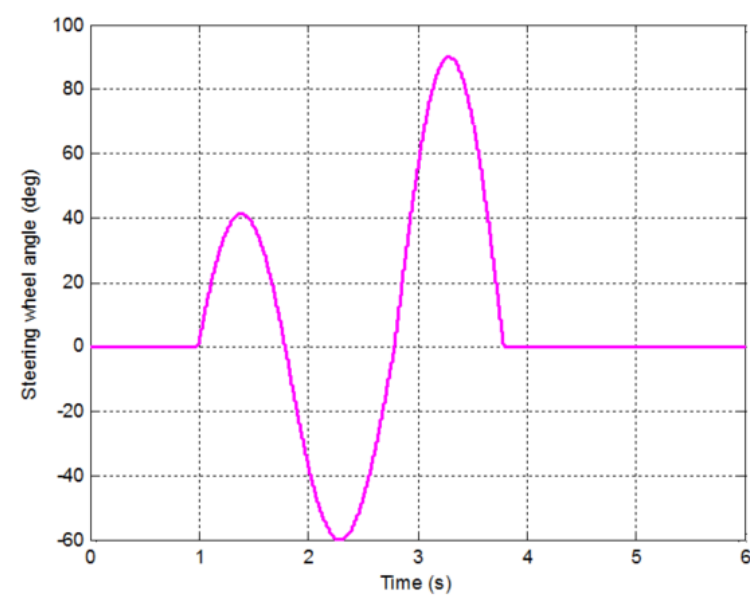

Fig. 9: Steering wheel angle input $(\delta=0$ and $\delta \neq 0)$.

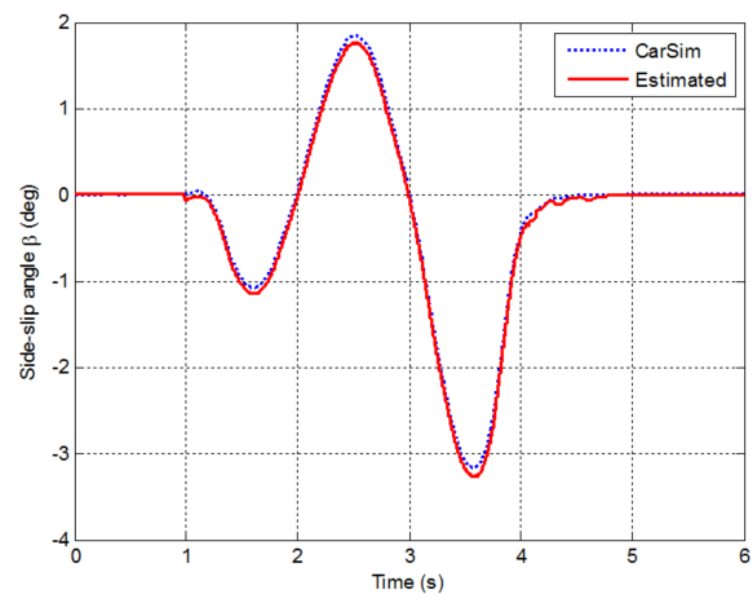

Fig. 10: Estimation of the vehicle side-slip angle for any condition of steering angle.

proved. Moreorver, the estimation results are favorable according to variable conditions of steering angle. In future studies, algorithms such as iterated EKF and adaptive iterated EKF can be examined to determine whether they yield excellent estimation results. Then, future research can consider their application for designing the control systems and hardware-in-the-loop simulations.

\section{References}

[1] LE, A. T. and C. K. CHEN. Vehicle stability control by using an adaptive slidingmode algorithm. Int. J. Vehicle Design. 2016, vol. 72 , iss. 2, pp. 107-131.

[2] MINGLI, S., C. LIANG, G. JIAHUA, and F. YONG. Estimation of vehicle side-slip angle using hybrid observer. In: proceedings of the 29th Chinese Control Conference $(C C C)$. China: 2010, pp. 5378-5383.

[3] LIMROTH., J. Real-time parameter estimation and adaptive stability control. USA, 2009. Ph.D. dissertation. Department of Mechanical Engineering, Clemson University.

[4] UNGOREN, A. Y. and H. PENG. A study on lateral speed estimation methods. Int. J. Vehicle Autonomous Systems. 2004, vol. 2, iss. $1 / 2$, pp. 126-144.

[5] DENG, W. and H. ZHANG. RLS-based online estimation on vehicle linear sideslip. In: proceedings of the American Control Conference. USA: 2006, pp. 3960-3965.

[6] ZARCHAN, P. and H. MUSOFF. Fundamentals of Kalman filtering: a practice approach, 3rd Ed. USA: American Institute of Aeronautics and Astronautics, 2009.

[7] KALMAN, R. E. A new approach to linear filtering and prediction problems. Trans ASME - J. Basic Engineering. 1960, Series $\mathrm{D}(82)$, pp. $35-45$.

[8] CHEN, B. C. and F. C. HSIEH. Side-slip angle estimation using extended Kalman filter. Vehicle System Dynamics. 2008, vol. 46 , iss. 1 , pp. $353-364$.

[9] CHEN, C. K. and A. T. LE. Vehicle sideslip angle and lateral force estimator based on extended Kalman filtering algorithm. Lecture Notes in Electrical Engineering. 2015, vol. 371, pp. 377-388.

[10] DEMERLY, J. D. and K. Y. TOUMI. Nonlinear analysis of vehicle dynamics (NAVDyn): A reduced order model for vehicle handling analysis. SAE International. ISBN 2000-01-1621. 
[11] RAJAMANI, R. Vehicle dynamics and control. In: Springer Science and Business Media. New York, USA: Springer, 2006.

[12] HEYDINGER, G. J., P. A. GRYGIER, and S. LEE. Pulse testing techniques applied to vehicle handling dynamics. SAE International. 1993, 930828.

[13] CHEN, C. K., T. K. DAO, and H. P. LIN. A compensated yaw-moment-based vehicle stability controller. Int. J. Vehicle Design. 2010, vol. 53, iss. 3 , pp. 220-238.

[14] FRANKLIN, G.-F. and J. D. POWELL. Digital control of dynamic systems, 3rd Ed. Addison Wesley Longman, 1998.

[15] DOUMIATI, M., A. CHARARA, A. VICTORINO, and D. LECHNE. Vehicle $d y$ namics estimation using Kalman filtering. ISTE Ltd and John Wiley and Sons, New York, 2013.

\section{About Authors}

Anh Tuan LE received his BS in Mechatronics Engineering from University of Technical Education and MS in Automation Engineering from University of Technology, Hochiminh City, Vietnam in 2006 and 2010, respectively, and $\mathrm{PhD}$ in Mechanical and Automatic Engineering from Da-Yeh University, Taiwan, in 2016. He is currently working as a lecturer in Faculty of Electrical and Electronics Engineering, Ton Duc Thang University, Ho Chi Minh City, Vietnam. His current research interests are process control and automation, robot dynamics, vehicle dynamics, vehicle stability control, antilock braking system, traction control system and control applications for vehicles and three-wheeled mobile robots.

\section{Appendix A Nomenclature}

$F_{x n}, F_{y n}$ : Longitudinal and lagged lateral tire forces.

$F_{z n}$ : Vertical force on each tire.

$B_{f}, B_{r}$ : Front and rear track widths.

$M$ : Vehicle mass.

$d$ : Offset from center of gravity (CG).

$I_{z}$ : Mass moment of inertia about the $\mathrm{z}$ axis.

$l$ : Wheelbase.

$r$ : Wheel radius.

$l_{f}, l_{r}$ : Distances from CG to front and rear axles.

$T_{b n}$ : Braking torque of each wheel.

$\delta$ : Steering angle.

$\delta_{w}$ : Steering wheel angle.

$\delta_{l}, \delta_{r}$ : Left and right steering angles.

$V_{x}, V_{y}$ : Vehicle longitudinal and lateral velocities.

$a_{x}, a_{y}$ : Longitudinal and lateral accelerations.

$\theta$ : Vehicle heading angle measured from $\mathrm{x}$ axis.

$\alpha_{n}$ : Slip angle of each tire.

$\gamma$ : Yaw rate.

$\beta$ : Side-slip angle.

$d$ : Offset from center of gravity (CG).

$i_{d n}$ : Longitudinal skid ratio of each tire.

$C_{d n}$ : Longitudinal tire stiffness during braking.

$C_{n}$ : Cornering stiffness of each tire.

$\mu$ : Tire-road frictional coefficient.

$V_{n}$ : Wheel ground contact point velocities.

$\omega_{n}$ : Wheel speeds.

"This is an Open Access article distributed under the terms of the Creative Commons Attribution License, which permits unrestricted use, distribution, and reproduction in any medium, provided the original work is properly cited (CC BY 4.0)." 